QCD Evolution Workshop 2013

International Journal of Modern Physics: Conference Series

Vol. 25 (2014) 1460037 (12 pages)

(C) The Authors

DOI: $10.1142 / \mathrm{S} 2010194514600374$

\title{
STUDIES OF ANALYTIC EVOLUTION OF TWO-PHOTON GENERALIZED DISTRIBUTION AMPLITUDE IN QCD*
}

\author{
ASLI TANDOGAN and ANATOLY V. RADYUSHKIN ${ }^{\dagger}$ \\ Physics Department, Old Dominion University, Norfolk, VA 23529, USA \\ and \\ Thomas Jefferson National Accelerator Facility, Newport News, VA 23606, USA
}

Published 2 January 2014

\begin{abstract}
We extend our method of analytic ERBL evolution for the case of distribution amplitudes that have jumps at some points $x=\zeta_{i}$ inside the support region $0<x<1$. As an application of the method, we use it for evolution of the two-photon generalized distribution amplitude. Our approach has advantages over the standard method of expansion in Gegenbauer polynomials, which requires infinite number of terms in order to accuretly reproduce functions in the vicinity of singular points, and over the method of straightforward iteration of initial distribution with evolution kernel which produces logarithmically divergent terms at each iteration. In our method, the logarithmic singularities are summed from the start, which immediately produces a continuous curve, with only one or two iterations needed afterwards in order to get precise results.
\end{abstract}

PACS numbers:12.38. $-\mathrm{t}, 11.10 .-\mathrm{z}$

\section{Introduction}

Construction of theoretical models for Generalized Parton Distributions (GPDs) $[1,2,3,4]$ is an inherent part of their studies. These models should satisfy several nontrivial requirements that follow from most general principles of quantum field theory. In this context, one could mention polynomiality, ${ }^{5}$ positivity, ${ }^{6-8}$ hermiticity, ${ }^{1}$ time reversal invariance, ${ }^{5}$ etc. These complicated conditions, however, are automatically satisfied by relevant Feynman diagrams of perturbation theory. In particular, analysis of simple one-loop diagrams is the basis of the factorized DD Ansatz [8] (FDDA) that is a standard element of codes generating models for GPDs.

In fact, the commonly used version of FDDA involves the assumption about universality of the DD profile function, which, though supported by one-loop examples,

\footnotetext{
*This is an Open Access article published by World Scientific Publishing Company. It is distributed under the terms of the Creative Commons Attribution 3.0 (CC-BY) License. Further distribution of this work is permitted, provided the original work is properly cited.

$\dagger$ Also at Bogoliubov Laboratory of Theoretical Physics, JINR, 141980 Dubna, Russian Federation.
} 
was not shown to be a mandatory property of double distributions. A possible way to go beyond the one-loop analysis, but still remain within the perturbation theory framework, is to incorporate pQCD evolution equations. Namely, the strategy is to take the expression for some one-loop diagram as the starting function for evolution, and use evolved patterns for modeling GPDs.

Implementation of such a program faces some technical difficulties. In particular, a well known property of GPDs $H(x, \xi)$ is that they are non-analytic at border points $x= \pm \xi$. For one-loop diagrams, this non-analyticity may take the form of cusps, jumps, and even delta-functions. Thus, one needs to develop methods of evolution for singular initial distributions. A simple example of a singular distribution is given by flat distribution amplitudes $\varphi(x)=$ const which do not vanish at the $x=0, x=1$ boundaries of the DA support region. In our paper [9], we described a method that allows to easily establish the major evolution pattern $(x \bar{x})^{t}$ for a flat DA, and provides an algorithm for an analytic calculation of corrections to it. The method was also applied to a DA $\varphi(x)=\operatorname{sgn}(x-1 / 2)$ that has a jump at $x=1 / 2$, in the middle of the support interval, with DA being antisymmetric with respect to that point.

In the present paper, we extend the method of Ref. [9] for studying evolution of generalized distribution amplitudes (GDAs) [10]. Similarly to GPDs, these functions are non-analytic at kinematics-dependent points $x=\zeta, 1-\zeta$ inside the support interval. The evolution of GPDs is further complicated by the fact that GPD evolution kernels also depend on skewness $\zeta$ (or $\xi$ ). Unlike GPDs, GDAs evolve according to the same $\zeta$-independent ERBL kernels as usual DAs, which allows us to concentrate on studying implications due to the non-analytic structure of the initial distribution. A particular object that we consider is the two-photon GDA [11] related to the reaction $\gamma^{*}(q) \gamma\left(q^{\prime}\right) \rightarrow \gamma\left(p_{1}\right) \gamma\left(p_{2}\right)$. In the QCD lowest order, it is proportional to the $V V \rightarrow V V$ ERBL evolution kernel, but the evolution of its $\ln Q^{2}$ derivative, in the leading logarithm approximation, is governed by the $q q \rightarrow q q$ ERBL kernel, just as in examples considered in Ref. [9].

\section{Evolution of Singular Distribution Amplitudes}

\subsection{Evolution equation: basics}

Evolution of the (logarithmic derivative of) nonsinglet two-photon GDAs in the leading logarithm approximation is governed by ERBL evolution equation ${ }^{12,13}$

$$
\frac{\partial \varphi(x, t)}{\partial t}=\int_{0}^{1}[V(x, y)]_{+} \varphi(y, t) d y
$$

where $t=2 C_{F} \ln \ln (\mu / \Lambda) / b_{0}$ is the leading logarithm QCD evolution parameter,

$$
V(x, y)=\left[\frac{x}{y}\left(1+\frac{1}{y-x}\right)\right] \theta(x<y)+\left[\frac{\bar{x}}{\bar{y}}\left(1+\frac{1}{x-y}\right)\right] \theta(y<x)
$$


is the evolution kernel (we use $\bar{x}=1-x$ and $\bar{y}=1-y$ ), which has singularity for $x=y$ regulated by the plus prescription

$$
[V(x, y)]_{+}=V(x, y)-\delta(y-x) \int_{0}^{1} V(z, y) d z
$$

with respect to the first argument $x$. In explicit form the evolution equation is

$$
\frac{\partial \varphi(x, t)}{\partial t}=\int_{0}^{1}[V(x, y) \varphi(y, t)-V(y, x) \varphi(x, t)] d y .
$$

The usual approach ${ }^{12,13}$ to solve ERBL equation is to expand initial DA over the eigenfunctions $x(1-x) C_{n}^{3 / 2}(2 x-1)$ of the evolution kernel. Each Gegenbauer projection then changes as a power $t^{-\lambda_{n}}$ of the evolution parameter. All anomalous dimensions $\lambda_{n}$ are positive, except for $\lambda_{0}$ which is zero, hence only the $\sim x(1-x)$ part survives for $t \rightarrow \infty$. For a pion DA given by a sum of a few Gegenbauer polynomials, this method gives a convenient analytic expression for the DA evolution. However, if the initial DA does not vanish at the end points, or has jumps inside the support region, one should formally take an infinite number of Gegenbauer polynomials. In practice, this means that one should sum over a very large number of terms to get a reasonably precise (point by point) result for the evolved DA.

Another way is to solve the evolution equation by iterations, i.e. write the solution as a series expansion $\varphi(x, t)=\sum_{n=0}^{\infty} t^{n} \varphi_{n}(x) / n$ !, with the functions $\varphi_{n}(x)$ satisfying the recurrence relation

$$
\varphi_{n+1}(x)=\int_{0}^{1}\left[V(x, y) \varphi_{n}(y)-V(y, x) \varphi_{n}(x)\right] d y .
$$

\subsection{Evolution of flat $D A$}

Taking flat DA $\varphi_{0}^{F}(x)=1$ as the simplest example of the function that does not vanish at the end points, one finds that the first iteration

$$
\varphi_{1}^{F}(x)=\int_{0}^{1}[V(x, y)-V(y, x)] d y=3 / 2+x \ln \bar{x}+\bar{x} \ln x \equiv v(x)
$$

has logarithmic singularities $\ln x$ and $\ln \bar{x}$ at the end points. They reflect the expansion of the $(x \bar{x})^{t}$ factor that appears in the all-order result, which is a regular function of $x$ with power behavior at the end points. The singular part of $\varphi_{1}^{F}(x)$ comes from the singular $1 /(x-y)$ part of the QCD evolution kernel, which brings $2+\ln (x \bar{x})$ into the iteration result.

Note that singularities of $V(x, y)$ and $V(y, x)$ at $x=y$ cancel each other in the integrand of evolution equation (4), even though the subtraction procedure in this integral does not look like a "+"-prescription with respect to the integration variable " $y$ ". But one can rewrite (4) as

$$
\frac{\partial \varphi(x, t)}{\partial t}=\int_{0}^{1} V(x, y)[\varphi(y, t)-\varphi(x, t)] d y+\varphi(x, t) \int_{0}^{1}[V(x, y)-V(y, x)] d y,
$$


where the first term has the desired structure of the "+"-prescription with respect to $y$. The integral in the second term coincides with the first iteration of the evolution kernel with the flat DA denoted in $(6)$ by $v(x)$. This evolution equation can be simplified through taking the Ansatz $\varphi(x, t)=e^{t v(x)} \Phi(x, t)$, resulting in

$$
\frac{\partial \Phi(x, t)}{\partial t}=\int_{0}^{1} V(x, y)\left[e^{t[v(y)-v(x)]} \Phi(y, t)-\Phi(x, t)\right] d y .
$$

Expanding $\Phi(x, t)=\sum_{n=0}^{\infty} t^{n} \Phi_{n}(x) / n$ ! , gives the recurrence relations

$$
\Phi_{n+1}(x)=\int_{0}^{1} V(x, y)\left[\sum_{l=0}^{n} \frac{n !}{(n-l) ! l !} \Phi_{l}(y)[v(y)-v(x)]^{n-l}-\Phi_{n}(x)\right] d y
$$

or, explicitly for the first terms,

$$
\begin{aligned}
& \Phi_{1}(x)=\int_{0}^{1} V(x, y)\left[\Phi_{0}(y)-\Phi_{0}(x)\right] d y \\
& \Phi_{2}(x)=\int_{0}^{1} V(x, y)\left[\Phi_{1}(y)-\Phi_{1}(x)+\Phi_{0}(y)[v(y)-v(x)]\right] d y
\end{aligned}
$$

etc. The first correction $\Phi_{1}(x)$ vanishes for the flat initial DA. Within this framework, the evolution of the flat distribution was studied in Ref. [9], where it was observed that the $t^{n}$ series converges rather rapidly as far as $t \lesssim 1$. When $t \gtrsim 1$, the DA is close to the asymptotic $x \bar{x}$ form, and one can switch to the solution in the form of Gegenbauer expansion.

\subsection{Evolution of jumps}

Another example of singularity is given by DAs with a jump, the simplest case being

$$
\Phi_{0}^{J}(x, \zeta ; a, b)= \begin{cases}a & 0<x \leqslant \zeta \\ b & \zeta<x<1 .\end{cases}
$$

The part of the first iteration $\Phi_{1}(x)$ generated by the singular part of the kernel

$$
\Phi_{1}^{J \operatorname{sing}}(x, \zeta ; a, b)=\left\{\begin{aligned}
(a-b) \ln \left[\frac{\zeta-x}{(1-x) \zeta}\right] & 0<x \leqslant \zeta \\
-(a-b) \ln \left[\frac{x-\zeta}{(1-\zeta) x}\right] & \zeta<x \leqslant 1
\end{aligned}\right.
$$

contains logarithmic terms $\ln |\zeta-x|$ singular for $x=\zeta$. Their structure may be understood in the following way. The original function $\varphi_{0}^{J}(x, \zeta ; a, b)$ may be represented as a sum of a constant $\frac{1}{2}(a+b)$ and a function $\frac{1}{2}(a-b) \operatorname{sgn}(\zeta-x)$ that jumps by $b-a$ at the point $x=\zeta$. The constant part has no singularities at $x=\zeta$, so one can apply the original $\frac{1}{2}(a+b)(x \bar{x})^{t} \Phi$ Ansatz to it, while for the jumping part one 
may use the Ansatz

$$
\begin{aligned}
\Phi^{J}(x, \zeta ; a, b ; t)= & \frac{a-b}{2} e^{2 t}(x \bar{x})^{t}\left[\left(\frac{1-x / \zeta}{1-x}\right)^{2 t} \theta(x<\zeta)\right. \\
& \left.+\left(\frac{1-\bar{x} / \bar{\zeta}}{1-\bar{x}}\right)^{2 t} \theta(x>\zeta)\right] \Phi(x, \zeta)+\Psi(x, \zeta, t) .
\end{aligned}
$$

The part containing square brackets is intended to take care of the evolution of the jump at $x=\zeta$. However, this part by construction vanishes at $x=\zeta$, while one would expect that evolution tends to convert $\varphi^{J}(x, \zeta ; a, b)$ into a universal $\zeta$-independent function proportional to $x \bar{x}$ or $x \bar{x}(1-2 x)$ (depending on the symmetry of the function). Thus, there should be also a part regular at the jump point. The function $\Psi(x, \zeta, t)$ is introduced to satisfy this requirement. It vanishes for $t=0$, but eventually becomes the dominant part.

Let us discuss a more general case, when a function has antisymmetric jumps at some locations $x=\zeta_{i}$. "Antisymmetric" means that the function approaches opposite values on the sides of a jump, so that "on average" it is zero at the jump points. Then one can try the Ansatz

$$
\varphi(x, t)=\Phi(x, t)+e^{t v(x)} \Psi(x, t)
$$

where $\Phi(x, t)=e^{t w(x)} \Phi_{0}(x)$, with the function $w(x) \sim \sum_{i} \ln \left|x-\zeta_{i}\right|$ intended to absorb major features of the evolution of the starting distribution $\varphi(x, t=0) \equiv \Phi_{0}(x)$ in the vicinity of the jump points, while the remainder $e^{t v(x)} \Psi(x, t)$ is expected to be a regular function vanishing for $t=0$. As a result, we get the following equation:

$$
\begin{aligned}
\frac{\partial \Psi(x, t)}{\partial t}= & \int_{0}^{1} V(x, y)\left[e^{t[v(y)-v(x)]} \Psi(y, t)-\Psi(x, t)\right] d y \\
& +e^{-t v(x)}\left\{\int_{0}^{1} V(x, y)[\Phi(y, t)-\Phi(x, t)] d y-\Delta w(x) \Phi(x, t)\right\},
\end{aligned}
$$

where $\Delta w(x) \equiv w(x)-v(x)$. This is an inhomogeneous evolution equation for $\Psi(x, t)$, with starting condition $\Psi(x, t=0)=0$. For its derivative at $t=0$ we have

$$
\left.\frac{\partial \Psi(x, t)}{\partial t}\right|_{t=0}=\int_{0}^{1} V(x, y)\left[\Phi_{0}(y)-\Phi_{0}(x)\right] d y-\Delta w(x) \Phi_{0}(x) \equiv \chi(x) .
$$

To avoid singularities at the jump points, we should adjust $w(x)$ in such a way as to make $\chi(x)$ a continuous function of $x$. Then $\Psi(x, t)=t \chi(x)+\ldots$, where the further terms of the $t$-expansion are given by second etc. iterations. 


\section{Structure of Photon Generalized Distribution Amplitude}

In the lowest QCD order, the non-singlet two-photon GDA is given by ${ }^{14}$

$$
\psi^{q}\left(x, \zeta, Q^{2}\right)=\frac{N_{C} e_{q}^{2}}{2 \pi^{2}} \log \frac{Q^{2}}{m^{2}} \varphi(x, \zeta),
$$

where the function $\varphi(x, \zeta)$ is proportional to the $V V \rightarrow V V$ component

$$
\begin{aligned}
\varphi(x, \zeta) & =\frac{\bar{x}(2 x-\zeta)}{\bar{\zeta}} \theta(x-\zeta)+\frac{\bar{x}(2 x-\bar{\zeta})}{\zeta} \theta(x-\bar{\zeta}) \\
& -\frac{x(2 \bar{x}-\bar{\zeta})}{\zeta} \theta(\zeta-x)-\frac{x(2 \bar{x}-\zeta)}{\bar{\zeta}} \theta(\bar{\zeta}-x)
\end{aligned}
$$

of the ERBL evolution kernel matrix. QCD corrections induce further evolution of the photon GDA. Namely, its derivative with respect to $\ln Q^{2}$ obeys ERBL evolution equation with the $q q \rightarrow q q$ kernel considered above. In what follows, we study ERBL evolution of the function $\varphi(x, \zeta, t)$ which for the starting evolution point $t=0$ coincides with $\varphi(x, \zeta)$.

The function $\varphi(x, \zeta)$ is antisymmetric with respect to $x \leftrightarrow \bar{x}$ interchange and symmetric with respect to $\zeta \leftrightarrow \bar{\zeta}$ interchange. Thus, without loss of generality, we may choose $\zeta \leq 1 / 2$. Then $0 \leq \zeta \leq \bar{\zeta} \leq 1$, and it makes sense to explicitly write the function in each of the three regions:

$$
\begin{aligned}
\varphi(x, \zeta)= & \left\{\frac{x}{\zeta \bar{\zeta}}\left[\bar{\zeta}^{2}+\zeta^{2}-2 \bar{x}\right] \theta(0 \leq x \leq \zeta)-\frac{\zeta(1-2 x)}{1-\zeta} \theta(\zeta \leq x \leq \bar{\zeta})\right. \\
& \left.-\frac{\bar{x}}{\zeta \bar{\zeta}}\left[\bar{\zeta}^{2}+\zeta^{2}-2 x\right] \theta(\bar{\zeta} \leq x \leq 1)\right\} .
\end{aligned}
$$

The function is discontinuous at $x=\zeta$ and $x=\bar{\zeta}$. Fig. 1 shows the $x$-profile of the two-photon GDA at different $\zeta$ values.

As $x$ approaches $\zeta$, the limiting value of the function from the left is $\varphi\left(\zeta_{-}, \zeta\right)=-1-\zeta \frac{1-2 \zeta}{1-\zeta}$, while from the right we have $\varphi\left(\zeta_{+}, \zeta\right)=-\zeta \frac{1-2 \zeta}{1-\zeta}$, so that the jump $\varphi\left(\zeta_{+}, \zeta\right)-\varphi\left(\zeta_{-}, \zeta\right)=1$ is equal to 1 .
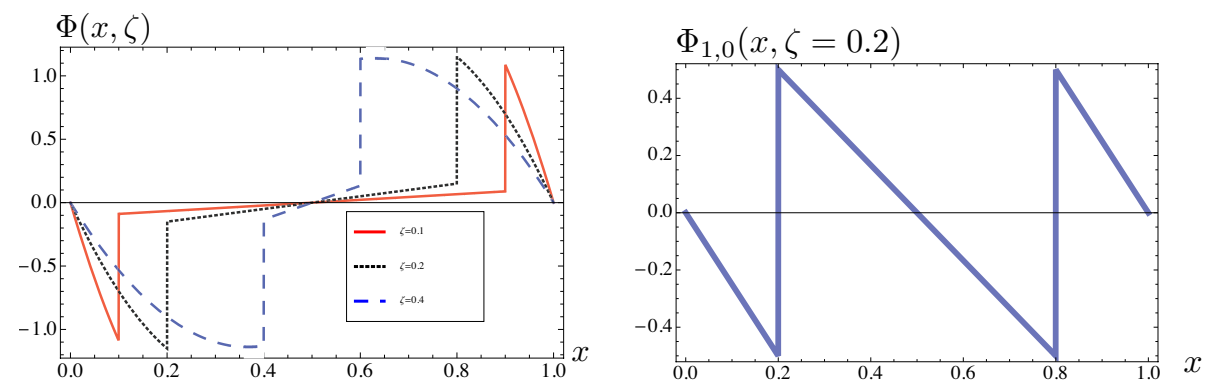

Fig. 1. Left: two-photon GDA profile function $\Phi(x, \zeta)$ at $\zeta=0.1 ; 0.2 ; 0.4$. Right: The $x$-profile of GDA $\Phi_{1,0}(x, \zeta)$ at $\zeta=0.2$ 
According to our discussion in the preceding section, to treat the evolution of a jump, we should represent the initial function as a sum of a function $\varphi_{2}(x, \zeta)$ continuous in the vicinity of each jump, and a function $\varphi_{1}(x, \zeta)$ that has an antisymmetric jump of necessary size. The function $\varphi_{1}(x, \zeta)$ will also specify the initial form of the $\Phi$-part of the evolution Ansatz (13) for this function, so we will denote it as $\Phi_{1,0}(x, \zeta)$. For simplicity, we will choose it to be given by linear functions of $x$ in each of the three regions. As a result,

$$
\Phi_{1,0}(x, \zeta)=-\frac{x}{2 \zeta} \theta(0<x<\zeta)+\frac{\bar{x}}{2 \zeta} \theta(\bar{\zeta}<x<1)+\frac{1-2 x}{2(1-2 \zeta)} \theta(\zeta<x<\bar{\zeta}) .
$$

The function $\Phi_{1,0}(x, \zeta)$ is discontinuous at $x=\zeta$ and $x=\bar{\zeta}$, see Fig. 1, right, where it is shown for $\zeta=0.2$. The function $\varphi_{2}(x, \zeta)$ specifying initial shape of the continuous part is obtained as the difference between $\varphi(x, \zeta)$ and $\Phi_{1,0}(x, \zeta)$.

\section{Evolution of the jump part of two-photon GDA}

Iteration of the initial function $\Phi_{1,0}(x, \zeta)$ with evolution kernel gives

$$
\begin{aligned}
\Phi_{1,1}(x, \zeta) \equiv & \int_{0}^{1} V(x, y)\left[\Phi_{1,0}(y, \zeta)-\Phi_{1,0}(x, \zeta)\right] d y \\
& =\frac{\theta(0<x<\zeta<1 / 2)}{2 \zeta(1-2 \zeta)}\{(1-2 \zeta)(1-4 \zeta) x+\zeta \bar{x} \ln \zeta+\bar{x}(1-3 \zeta) \ln \bar{\zeta} \\
& -2 \zeta(1-2 \zeta)[\ln (\zeta-x)+\ln (\bar{\zeta}-x)] \\
& -2(1-4 \zeta)[(x-\zeta) \ln (\zeta-x)+(\bar{\zeta}-x) \ln (\bar{\zeta}-x)] \\
& +(1-2 \zeta)[x \bar{x} \ln x+(1-x \bar{x}) \ln \bar{x}]\}-\{x \rightarrow \bar{x}\} \\
& +\frac{\theta(\zeta<x<\bar{\zeta})\{\zeta(1-4 \zeta)(1-2 x)+(1-2 x)(1-3 \zeta) \ln \bar{\zeta}}{2 \zeta(1-2 \zeta)}\{ \\
& +2 \zeta(1-2 \zeta)[\ln (x-\zeta)-\ln (\bar{\zeta}-x)] \\
& +(1-4 \zeta)[(x-\zeta) \ln (x-\zeta)-(\bar{\zeta}-x) \ln (\bar{\zeta}-x)] \\
& \left.+(1-4 \zeta)(\bar{x} \ln \bar{x}-x \ln x)+\zeta\left(x^{2}+\bar{x}^{2}\right)(\ln \bar{x}-\ln x)\right\}
\end{aligned}
$$

As expected, $\Phi_{1,1}(x, \zeta)$ has logarithmic singularities

$$
-\ln (\zeta-x) \theta(x<\zeta)+\ln (x-\zeta) \theta\left(\zeta<x<\frac{1}{2}\right)-\{x \rightarrow \bar{x}\}
$$

for $x=\zeta$ and $x=\bar{\zeta}$ (see Fig. 2). The sum of these terms may be written as $2(\ln |x-\zeta|+\ln |x-\bar{\zeta}|) \Phi_{1}(x, \zeta)$ plus regular terms, which suggests to take the Ansatz (13) with $w(x)$ containing $2(\ln |x-\zeta|+\ln |x-\bar{\zeta}|)$. Namely, let us try the function $w_{0}(x, \zeta)$ given by

$$
w_{0}(x, \zeta)=v(x)+4+2 \ln |x-\zeta|+2 \ln |x-\bar{\zeta}| .
$$

The constant part " 4 " was chosen to make the integral of $\Delta w_{0}(x) \equiv w_{0}(x)-v(x)$ closer to zero (it vanishes both for $\zeta=0$ and $\zeta=1$ ), i.e. to keep the overall 


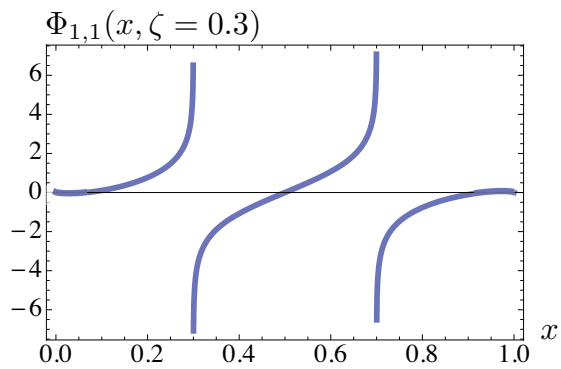

Fig. 2. First iteration $\Phi_{1,1}(x, \zeta)$ for $\zeta=0.3$.
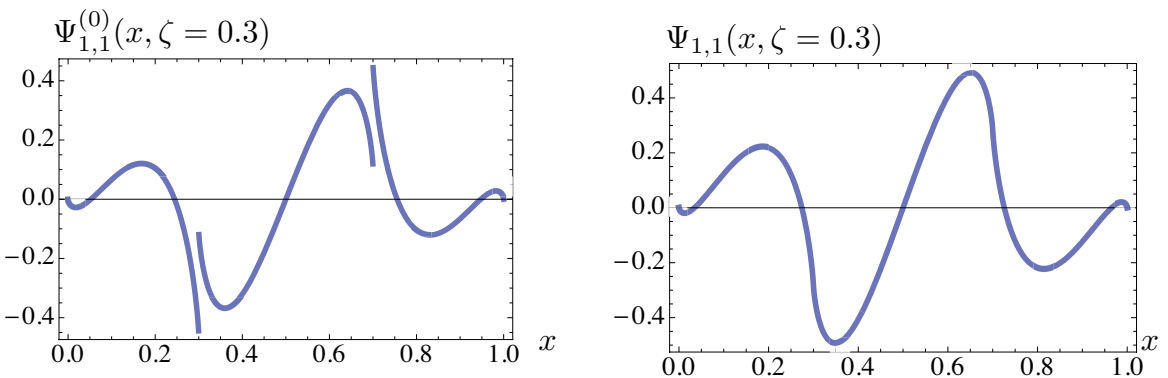

Fig. 3. Correction functions $\Psi_{1,1}^{(0)}(x, \zeta=0.3)$ (with $w_{0}$ Ansatz, left) and $\Psi_{1,1}(x, \zeta=0.3$ ) (with modified Ansatz, right).

normalization of the Ansatz factor closer to 1. Resulting function (which gives the first term of the $\Psi$-part of the Ansatz (13)) is given by

$$
\Psi_{1,1}^{(0)}(x, \zeta)=\Phi_{1,1}(x, \zeta)-\Delta w_{0}(x, \zeta) \Phi_{1}(x, \zeta)
$$

and shown in Fig. 3, left. One can see that, after the subtraction of singularities, we still have finite jumps for $x=\zeta$ and $x=\bar{\zeta}$. Explicit calculation gives

$$
\begin{aligned}
\Psi_{1,1}^{(0)}\left(\zeta_{+}, \zeta\right)-\Psi_{1,1}^{(0)}\left(\zeta_{-}, \zeta\right) & =4+2 \ln (|1-2 \zeta|)+(2-\zeta) \ln \zeta+(2-\bar{\zeta}) \ln \bar{\zeta} \\
& \equiv-w_{1}(\zeta)
\end{aligned}
$$

Thus, if we add $w_{1}(\zeta) \Phi_{1,0}(x, \zeta)$ to $\Psi_{1,1}^{(0)}(x, \zeta)$, we obtain the correction function $\Psi_{1,1}(x, \zeta)$ that is continuous at the border points $x=\zeta$ and $x=\bar{\zeta}$ (see Fig. 3, right). This corresponds to the following $\Phi$-part of the Ansatz (13)

$$
\Phi_{1}(x, \zeta, t)=e^{t v(x)}\left(\frac{|1-x / \zeta||1-x / \bar{\zeta}|}{|1-2 \zeta|}\right)^{2 t} \zeta^{t \zeta} \bar{\zeta}^{t} \bar{\zeta} \Phi_{1,0}(x, \zeta) .
$$

for the function $\varphi_{1}(x, \zeta, t)$. The function $\Phi_{1}(x, \zeta, t)$ is illustrated in Fig. 4. 


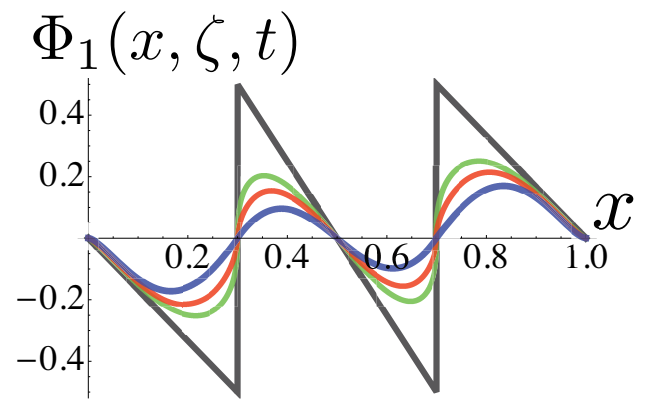

Fig. 4. Ansatz function $\Phi_{1}(x, \zeta=0.3, t)$ for $t=0,0.2,0.3,0.5$.

According to Ansatz (13), after fixing the function $w(x)$ from the requirement of continuity of $\Psi_{1,1}(x, \zeta)$, one should deal with the evolution equation (15) for the $\Psi$-part of the Ansatz. This equation specifies that $\partial \Psi_{1}(x, \zeta, t) / \partial t$ for $t=0$ is given by $\Psi_{1,1}(x, \zeta)$. Thus, for small $t$, we can approximate $\Psi_{1}(x, \zeta, t)$ by $t \Psi_{1,1}(x, \zeta)$. As one can see from Fig. 4, left, the correction due to the $\Psi$ term is rather small for $t=0.2$. It just reduces somewhat the amplitude of oscillations.

However, the correction becomes more and more visible with growing $t$, see Fig. 4, right, where the evolved function is shown for $t=0.4$ with and without the first $\Psi$-type correction term included. The total function is now clearly nonzero at the "border" points $x=\zeta$ and $x-1-\zeta$. This is because $\Psi_{1,1}(x, \zeta)$ is nonzero at these points. As we discussed, the $\Psi$-part becomes dominant for large $t$ and brings the shape of $\varphi_{1}(x, \zeta, t)$ to the asymptotic form $x \bar{x}(2 x-1)$ of the antisymmetric DAs. We can see that, for $t=0.4$ already, the total function strongly resembles the asymptotic shape $x \bar{x}(2 x-1)$. However, for such large $t$ values the simplest linear- $t$ approximation for $\Psi(x, \zeta, t)$ is too crude, and one should go beyond the first iteration. The work in this direction is now in progress.

\section{Evolution of cusp part of two-photon GDA and total result}

In this section, we study the evolution of the second function, namely $\varphi_{2}(x, \zeta)$. Its initial form $\Phi_{2,0}(x, \zeta)$ is continuous for $x=\zeta$ and $x=\bar{\zeta}$ and is given by

$$
\begin{aligned}
\Phi_{2,0}(x, \zeta)= & -\left(\frac{x}{\zeta}\right) \frac{1+\zeta-4 \zeta^{2}+4(\zeta-x)}{2(1-\zeta)} \theta(\zeta-x)-\{x \rightarrow 1-x\} \\
& -\left(\frac{1-2 x}{1-2 \zeta}\right) \frac{1+\zeta-4 \zeta^{2}}{2(1-\zeta)} \theta(x-\zeta) \theta(\bar{\zeta}-x)
\end{aligned}
$$

see Fig. 6 for a graphical representation. Since $\Phi_{2,0}(x, \zeta)$ is a continuous function vanishing at the end points, the easiest way to get its evolution is to use straightforward $t^{n}$ expansion with coefficients given by successive iterations of the evolution 


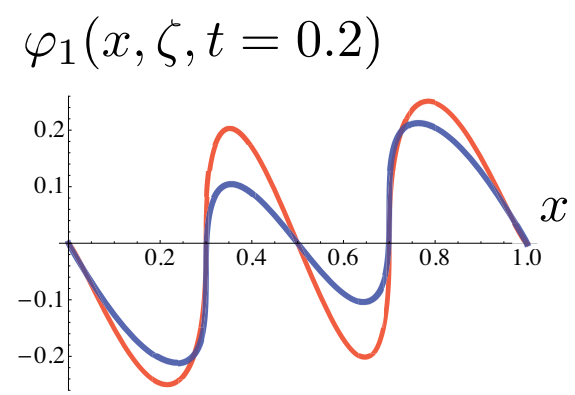

$$
\varphi_{1}(x, \zeta, t=0.4)
$$

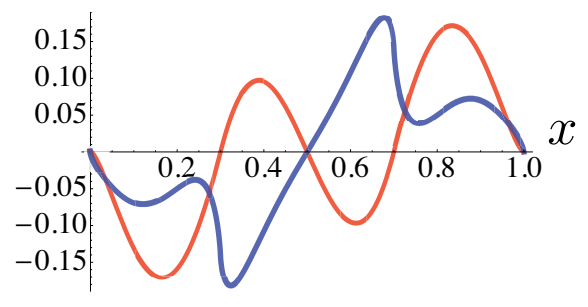

Fig. 5. Effect of inclusion of $\Psi_{1,1}(x, \zeta)$ correction for $t=0.2$ (left) and $t=0.4$ (right); $\zeta=0.3$ in both cases.
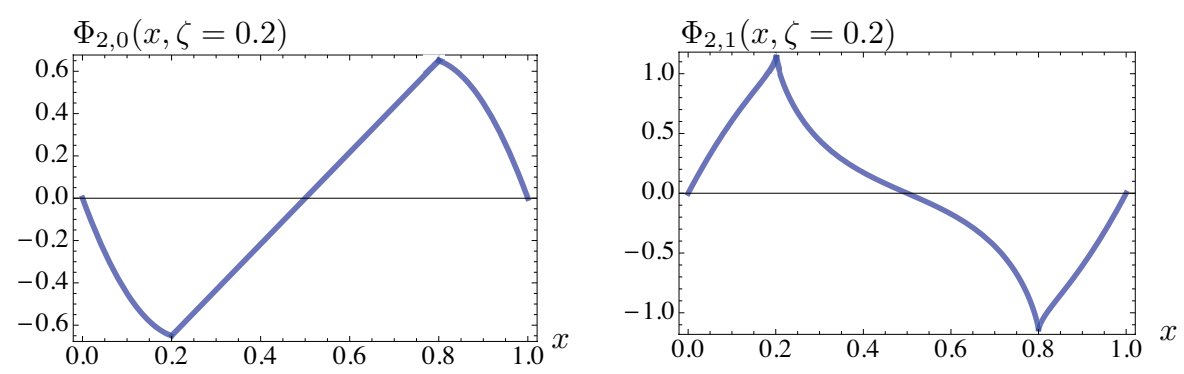

Fig. 6. Left: Initial function $\Phi_{2,0}(x, \zeta)$ for $\zeta=0.2$. Right: First iteration function $\Phi_{2,1}(x, \zeta=0.2)$.

kernel with $\Phi_{2,0}(x, \zeta)$. The result of the direct calculation of the first expansion component is

$$
\begin{aligned}
\Phi_{2,1}(x, \zeta)= & \frac{\theta(0<x<\zeta)}{2 \zeta \bar{\zeta}(1-2 \zeta)}\left\{x(1-2 \zeta)\left[1+9 \zeta-8 \zeta^{2}-6 x\right]\right. \\
& -x \bar{x}(1-2 \zeta)\left[1+\zeta-4 \zeta^{2}+4(\zeta-x)-4\right] \ln \bar{x} \\
& -\bar{x}\left[1+\zeta-4 \zeta^{2}\right] \zeta \ln \zeta+\bar{x}\left[1+\zeta-4 \bar{\zeta}^{2}\right] \bar{\zeta} \ln \bar{\zeta} \\
& +x \bar{x}(1-2 \zeta)\left[1+\zeta-4 \zeta^{2}+4(\zeta-x)\right] \ln x \\
& -(1-2 \zeta)\left[1+\zeta-4 \zeta^{2}-4 \bar{\zeta}+8 x \bar{x}\right] \ln [(1-\zeta / \bar{x}] \\
& \left.-\left[1+\zeta-4 \zeta^{2}-4 x(1-2 \zeta)\right](\zeta-x) \ln \left[\frac{\bar{\zeta}-x}{\zeta-x}\right]\right\}+ \\
& \frac{\theta(\zeta<x<1 / 2)}{2 \zeta \bar{\zeta}(1-2 \zeta)}\left\{(1-2 x) \zeta\left[1+\zeta-4 \zeta^{2}+2 \zeta(1-2 \zeta)\right]\right. \\
& +(1-2 x) \bar{\zeta}\left[1+\zeta-4 \bar{\zeta}^{2}\right] \ln \bar{\zeta}-2 x \bar{x} \zeta\left[1+\zeta-4 \zeta^{2}\right] \ln [x / \bar{x}]
\end{aligned}
$$



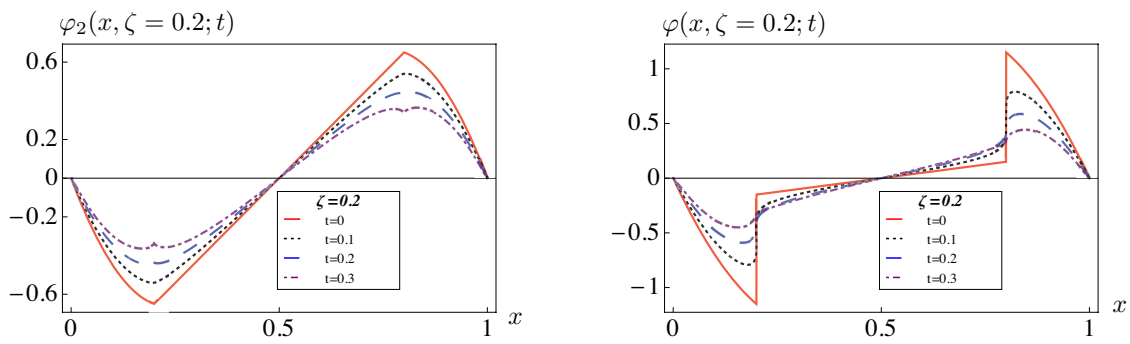

Fig. 7. Left:Evolution of GDA $\Phi_{2}(x, \zeta, t)$ at $t=0, t=0.2, t=0.3$ and $t=0.5$. Right: Evolution of total GDA $\Phi(x, \zeta, t)$ at $t=0, t=0.1, t=0.2$ and $t=0.3$

$$
\begin{aligned}
& -\left[1+\zeta-4 \zeta^{2}-4 \bar{x}(1-2 \zeta)\right](\bar{x}-\zeta) \ln [(1-\zeta / \bar{x}] \\
& \left.+\left[1+\zeta-4 \zeta^{2}-4 x(1-2 \zeta)\right](x-\zeta) \ln [1-\zeta / x]\right\} \\
& -\{x \rightarrow \bar{x}\}+v(x) \Phi_{2,0}(x, \zeta) .
\end{aligned}
$$

Here, as usual, $v(x)$ is $3 / 2+x \ln \bar{x}+\bar{x} \ln x$. The function $\Phi_{2,1}(x, \zeta=0.2)$ is shown in Fig. 6, right. One can see that it is continuous at points $x=\zeta$ and $x=\bar{\zeta}$. The evolution of $\varphi_{2}(x, \zeta ; t)$ generated by the first correction is illustrated by Fig. 7 , left. Adding the result for $\varphi_{1}(x, \zeta, t)$ obtained in previous sections, we end up with the evolution of the total function $\varphi(x, \zeta, t)$ illustrated in Fig. 7, right.

\section{Summary}

We extended the method for performing analytic ERBL evolution developed in Ref. [9] to make it applicable for functions that have jumps at some points $x=\zeta_{i}$ inside the support region $0<x<1$ of distribution amplitudes. As an illustration of the method, we applied it for studying the evolution of two-photon GDA. Unlike the standard method of expansion in Gegenbauer polynomials, which requires infinite number of terms in order to eliminate singularities of initial distributions, our method needs only one or two iterations in order to get a reliable and continuous result. Another advantage is that our method gives the results in analytic form.

\section{Acknowledgements}

Authored by Jefferson Science Associates, LLC under U.S. DOE Contract No. DEAC05-06OR23177. The U.S. Government retains a non-exclusive, paid-up, irrevocable, world-wide license to publish or reproduce this manuscript for U.S. Government purposes. 


\section{References}

1. D. Mueller, D. Robaschik, B. Geyer, F. M. Dittes and J. Horejsi, Fortschr. Phys. 42, p. 101 (1994).

2. X.-D. Ji, Phys. Rev. Lett. 78, 610 (1997).

3. A. V. Radyushkin, Phys. Lett. B380, 417 (1996).

4. J. C. Collins, L. Frankfurt and M. Strikman, Phys. Rev. D56, 2982 (1997).

5. X.-D. Ji, J. Phys. G24, 1181 (1998).

6. A. D. Martin and M. G. Ryskin, Phys. Rev. D57, 6692 (1998).

7. B. Pire, J. Soffer and O. Teryaev, Eur. Phys. J. C8, 103 (1999).

8. A. V. Radyushkin, Phys. Rev. D59, p. 014030 (1999).

9. A. Tandogan and A. V. Radyushkin, Int.J.Mod.Phys.Conf.Ser. 04, 227 (2011).

10. M. Diehl, T. Gousset, B. Pire and O. Teryaev, Phys.Rev.Lett. 81, 1782 (1998).

11. B. Pire and L. Szymanowski, Phys.Lett. B556, 129 (2003).

12. A. Efremov and A. Radyushkin, Phys.Lett. B94, 245 (1980).

13. G. P. Lepage and S. J. Brodsky, Phys.Rev. D22, p. 2157 (1980).

14. M. El Beiyad, B. Pire, L. Szymanowski and S. Wallon, Phys.Rev. D78, p. 034009 (2008). 